\title{
Determination of Sedimentary Basin Basement Depth: A Space Domain Based Gravity Inversion using Exponential Density Function
}

\author{
Vishnubhotla CHAKRAVARTHI and Batta RAMAMMA \\ Centre for Earth and Space Sciences, \\ University of Hyderabad, Hyderabad, India; \\ e-mails: vcvarthi@rediffmail.com (corresponding author), \\ ramageophd@gmail.com
}

\begin{abstract}
An automatic inversion using ridge regression algorithm is developed in the space domain to analyze the gravity anomalies of sedimentary basins, among which the density contrast decreases with depth following a prescribed exponential function. A stack of vertical prisms having equal widths, whose depths become the unknown parameters to be estimated, describes the geometry of a sedimentary basin above the basement complex. Because no closed form analytical equation can be derivable in the space domain using the exponential density-depth function, a combination of analytical and numerical approaches is used to realize forward gravity modeling. The depth estimates of sedimentbasement interface are initiated and subsequently improved iteratively by minimizing the objective function between the observed and modeled gravity anomalies within the specified convergence criteria. Two gravity anomaly profiles, one synthetic and a real, are interpreted using the proposed technique to demonstrate its applicability.
\end{abstract}

Key words: gravity anomaly, exponential density contrast, automatic inversion, space domain, sedimentary basin. 


\section{INTRODUCTION}

Gravity method plays an important role in the studies related to sedimentary basin modeling because detectable gravity anomalies can be observable on the surface of the Earth due to the presence of significant density contrast between sediment infill and the underlying basement. These observed gravity anomalies, considered to have been made on topographic elevations, can be modeled to decipher the geometry of the basement structure below the sedimentary load. Due to deficit in density of sedimentary rocks with the underlying basement complex, negative gravity anomalies are usually observed over sedimentary basins. It is well-known that the interpretation of gravity anomalies for subsurface density structure(s) is a non-unique problem, because the surface gravity anomalies can be explained by a variety of mass distributions at different depths (Blakely 1995). Such an ambiguity in gravity interpretation is often tackled by assigning a mathematical geometry to the anomalous mass with a known density and then to invert the anomalies for the unknown parameters such that the estimated structure is geologically sensible. Using a priori information in model space (derived from drilling/ other geophysical data) would further reduce the degree of uncertainty in interpretation.

Many 2D indirect methods (forward modeling) are available to compute the gravity anomalies of geological sources with uniform density (e.g., Won and Bevis 1987, Singh 2002). However, these forward modeling techniques find limited application in analyzing gravity anomalies of sedimentary basins because the parameters describing the structure are not known in advance. Bott (1960) and Murthy and Rao (1989) proposed direct methods of interpretation to solve the structures of sedimentary basins from the observed gravity anomalies, where the cross-section of a sedimentary basin was viewed as a collage of vertical prisms, all having equal widths and uniform density. However, the assumption of uniform density for sedimentary rocks is seldom valid in reality (Abdoh et al. 1990, Abbott and Louie 2000, Chakravarthi 2003, Gómez-Ortiz et al. 2005, Mantlík and Matias 2010, Kadima et al. 2011). Cowie and Karner (1990) demonstrated that the density-depth curves constructed for different stratigraphic units in sedimentary basins from measured well density logs exhibit a range of densities but the mean sediment density clearly increases with depth with the highest rate of increase in the top few kilometers. Such a variation of density with depth can be effectively simulated by an exponential density function if differential compaction is assumed to be the most important diagenetic process in the evolution of sedimentary basins (Cordell 1973, García-Abdeslem 1992). Hence, the use of exponential density-depth function in the analysis of gravity anomalies of sedimentary basins often paves the way for more reliable interpreta- 
tions. However, it becomes a strenuous task to accommodate exponential density variation in the existing forward modeling schemes, including some of the available commercial software such as GM-SYS (Northwest Geophysical Associates 2004), because large numbers of constant density bodies are required to adequately explain the same density structure (Zhou 2013).

Direct modeling methods are being developed to analyze the gravity anomalies of sedimentary basins using the exponential density-depth function. Because of the fact that no closed form analytical equations can be derivable in the space domain for the gravity anomalies with an exponential density function, many algorithms perform forward modeling in the frequency domain and then transform the anomalies back to the space domain for further analysis. For instance, Cordell (1973) developed a recursive method made use of both the gravity field and its vertical derivative (determined by convolution in discrete Fourier series) to solve the structure of a sedimentary basin from observed gravity anomalies. Granser (1987) had calculated the gravity effect of a structure based on series expansion, the numerical evaluation of which was performed by fast Fourier transform. Chai and Hinze (1988) proposed methods to analyze both profile and two-dimensional gravity data, where the forward modeling was realized in the wave number domain followed by its conversion to the space domain by a shift-sampling technique. Rao et al. (1993) derived Fourier transforms of gravity anomalies of some simple geometric models with exponential density contrast and used them in the analysis of the gravity anomalies of sedimentary basins; however, these strategies likely to yield unreliable interpretations when the sediment-basement interface has major undulations as in the case of the San Jacinto graben, California. The method developed by Rao and Rao (1999) also involved the calculation of the gravity effect in the frequency domain and its subsequent transformation to the space domain by Filon's method (Filon 1928). In recent past, Chappell and Kusznir (2008) extended the method of Granser (1987) to calculate the gravity anomaly as a function of the Fourier transforms of the bounding surfaces of a basin with irregular top and bottom surfaces. Nonetheless, the enlisted methods incur truncation errors when the modeled anomalies transform from the frequency domain to the space domain (Chakravarthi and Sundararajan 2007).

On the other hand, a few space domain based algorithms are available to model the gravity anomalies, where the exponential density variation was accommodated in the interpretation by alternative means. For instance, Murthy and Rao (1979) proposed the subdivision of each side of a 2D polygon into a number of segments, along which the density contrast was assumed to vary linearly with depth, whereas Guspi (1990) described the exponential density-depth function by a series approximation to compute the gravity response. The method of Murthy and Rao (1979) consumes signifi- 
cant amount of time even for forward modeling (Rao et al. 1994), whereas the method of Guspi (1990) requires the knowledge of the degree of the polynomial, which is generally not known a priori.

In this paper, we develop a space domain based inversion technique to analyze the gravity anomalies of sedimentary basins, among which the density contrast varies exponentially with depth. The present technique uses ridge regression algorithm to analyze the gravity anomalies. The applicability of the method is exemplified with both synthetic and real field examples.

\section{FORWARD MODELING - THEORETICAL CONSIDERATIONS}

Figure 1 shows the cross-section of a sedimentary basin. Let the profile, $\mathrm{AA}^{\prime}$, run along the $x$-axis transverse to the strike of the basin. The structure of the basin is approximated by a series of outcropping vertical prisms put in juxtaposition and having equal widths. In a Cartesian coordinate system, let $2 T$ be the width of one such prism along the $x$-axis and $z_{B}$ represent the thickness of the corresponding prism along the $z$-axis, positive vertically downwards (Fig. 1). Let the density contrast along the prism vary vertically with depth following the exponential equation of the form (Cordell 1973)

$$
\Delta \rho(z)=\Delta \rho_{0} e^{-\lambda z},
$$

where $\Delta \rho(z)$ is the density contrast at any depth $z, \Delta \rho_{0}$ is the density contrast observed at the ground surface, $\lambda$ is a constant expressed in inverse length units. The gravity anomaly of one such prism, $\Delta g_{\text {prm }}\left(x_{j}, z_{j}\right)$, at any observation, $P\left(x_{j}, z_{j}\right)$, on the profile, $\mathrm{AA}^{\prime}$, outside the source region can be expressed as

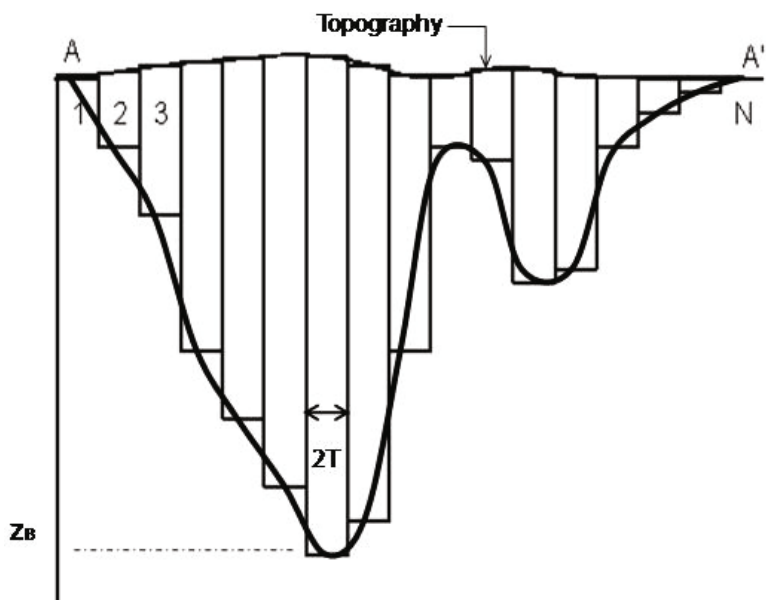

Fig. 1. Cross-section of a sedimentary basin (curved line) and its approximation by an ensemble of vertical prisms (step line). 


$$
\Delta g_{\mathrm{prm}}\left(x_{j}, z_{j}\right)=2 G \int_{s} \Delta \rho(z) \frac{\left(z-z_{j}\right) d x d z}{\left[{\overline{x-x_{j}}}^{2}+{\overline{z-z_{j}}}^{2}\right]} .
$$

Here, $G$ is universal gravitational constant and $(x, z)$ are source coordinates. Substituting Eq. 1 for $\Delta \rho(z)$ in Eq. 2 and upon integration within the limits of $x$, Eq. 2 takes the form

$$
\Delta g_{\text {prm }}\left(x_{j}, z_{j}\right)=2 G \Delta \rho_{0} \int_{0}^{z_{b}} e^{-\lambda z}\left[\tan ^{-1} \frac{\left(\overline{x-x_{j}}+T\right)}{\overline{z-z_{j}}}-\tan ^{-1} \frac{\left(\overline{x-x_{j}}-T\right)}{\overline{z-z_{j}}}\right] d z \text {. }
$$

Equation 3 has to be solved numerically because no closed form solution exists for it in the space domain. Further, it is to be realized that Eq. 3 is strictly valid for the profile, $\mathrm{AB}$, which runs transverse to the strike of the prism. In case the profile runs at an angle, $\alpha$, with the $x$-axis then $x_{j}$ in Eq. 3 needs to be replaced by $x_{j} \cos \alpha$ (Chakravarthi and Ramamma 2013).

The total gravity anomaly produced by a basin at any observation can be obtained as

$$
\Delta g_{\text {basin }}\left(x_{j}, z_{j}\right)=\sum_{i=1}^{N} \Delta g_{\text {prm }}\left(x_{i}, z_{i}\right),
$$

where $N$ stands for the number of prisms/observations on the profile.

\section{INVERSION OF GRAVITY ANOMALIES}

The objective of gravity inversion is to fit the modeled gravity anomalies to the observed ones by adjusting the thickness parameters of the basin in a least square sense such that the modeled gravity response of the optimum depth structure mimics the observed anomaly. The present algorithm consists of two modules, one for initialization of the model space and the other for refinement. The algorithm performs both modules automatically in the sense that it initiates the depth structure of a sedimentary basin from observed gravity anomalies and improves the structure iteratively, based on the differences between the observed and modeled gravity anomalies within the specified convergence criteria.

For initialization, it is presumed that the observed gravity anomaly at each station on the profile is being generated by an infinite horizontal subsurface slab in which the density contrast decreases exponentially with depth following Eq. 1. Accordingly, initial or approximate depths to basement interface are estimated using the Bouguer slab formula of Cordell (1973)

$$
z_{\text {Bou }}=\frac{-1}{\lambda} \log \left(1-\frac{\lambda \Delta g_{\text {obs }}\left(x_{i}, z_{i}\right)}{2 \pi G \Delta \rho_{0}}\right),
$$

where $\Delta g_{\text {obs }}\left(x_{i}, z_{i}\right)$ is the observed residual gravity anomaly at any station. 
The modeled gravity response of the basin, $\Delta g_{\text {basin }}\left(x_{j}, z_{j}\right)$, calculated at each observation on the profile using Eq. 4 obviously deviates from the observed anomaly, $\Delta g_{\text {obs }}\left(x_{j}, z_{j}\right)$, because the initial depths obtained from Eq. 5 are only approximate. The difference between these two anomalies at any observation at the end of the $k$-th iteration can be expressed as the cumulative of the products of vertical gradients of the anomaly and corresponding depth improvements of the prisms as

$$
\Delta g_{\text {obs }}\left(x_{j}, z_{j}\right)-\Delta g_{\text {basin }}\left(x_{j}, z_{j}\right)=\sum_{i=1}^{N}\left[\frac{\partial \Delta g_{\text {prm }}\left(x_{i}, z_{i}\right)}{\partial z}\right]_{z_{k}} d z_{i} .
$$

Linear equation similar to Eq. 6 is constructed for each observation and $N$ normal equations are framed and solved for the improvements in $N$ depth parameters of the prisms by minimizing the objective function $J$, defined as the sum of the squares of the differences between the observed and modeled gravity anomalies,

$$
J=\sum_{j=1}^{N}\left[\Delta g_{\text {obs }}\left(x_{j}, z_{j}\right)-\Delta g_{\text {basin }}\left(x_{j}, z_{j}\right)\right]^{2} .
$$

The system of normal equations can be expressed in a matrix form as

$$
(A+\delta I) X=B,
$$

where $A$ is a $n x n$ matrix whose elements $A_{n j}$ are given by

$$
\begin{gathered}
A_{n j^{\prime}}=\sum_{n=1}^{N} \sum_{m=1}^{N} \frac{\partial \Delta g_{\mathrm{prm}}\left(x_{m}, z_{m}\right)}{\partial a_{j^{\prime}}} \frac{\partial \Delta g_{\mathrm{prm}}\left(x_{m}, z_{m}\right)}{\partial a_{n}}, \quad j^{\prime}=1,2, \ldots, N, \\
X=d a_{n}, \\
B=\sum_{m=1}^{N}\left[\Delta g_{\text {obs }}\left(x_{m}, z_{m}\right)-\Delta g_{\text {basin }}\left(x_{m}, z_{m}\right)\right] \frac{\partial \Delta g_{\text {prm }}\left(x_{m}, z_{m}\right)}{\partial a_{j^{\prime}}}, \quad j^{\prime}=1,2, \ldots, N,
\end{gathered}
$$

Here, $a_{n}, n=1,2, \ldots, N$ are depth parameters of prisms and $d a_{n}$ represents corresponding depth improvements. $\delta$ is the damping factor, and $I$ is a diagonal matrix containing the diagonal elements of the matrix $A$. The partial derivatives required in Eqs. 9-10 are evaluated numerically, which involves the calculation of the rate of change of the gravity anomaly with respect to the thickness of each prism. Initially the value of $\delta$ is set to an arbitrary value of 0.5 and Eq. 8 is solved for the increments/decrements $d a_{n}$ and subsequently added to/subtracted from existing parameters of $a_{n}$ to obtain the improved parameters, $a_{n}^{\prime}$. If the current value of the objective function, $J_{\text {mod, }}$, obtained with the improved parameters, $a_{n}^{\prime}$, is less than its previous value, $J$, then $J_{\text {mod }}$ is assigned to $J$ and $a_{n}^{\prime}$ to $a_{n}$, and the damping factor, $\delta$, is de- 
creased by a factor of $1 / 2$. In case $J_{\bmod }$ is greater than $J$, then the value of $\delta$ is doubled and Eq. 8 is solved for $d a_{n}$ and are added to/subtracted from $a_{n}$ and this process repeats until $J_{\text {mod }}$ becomes less than or equal to $J$. The algorithm terminates in case:

$\checkmark$ the specified number of iterations are completed or,

$\square$ the current value of the objective function $J$ falls below user specified predefined allowable error or,

$\checkmark$ the current value of $\delta$ attains a large value.

\section{APPLICATIONS}

The applicability of the proposed inversion technique is demonstrated on two examples: one synthetic and a real. In case of real field example, the observed gravity anomalies across the San Jacinto graben, California, are analyzed and compared with those previously reported. In both cases the density contrast varies exponentially with depth and observer locations are at the top of the topography at $z_{j}=0$.

\subsection{Synthetic example}

Figure 2a shows 40 equispaced noisy gravity anomalies (solid circles) in the interval $x \in[0 \mathrm{~km}, 40 \mathrm{~km}]$ produced by a synthetic model, whose structure resembles a typical block faulted intracratonic rift basin filled with thick sectioned sediments (Fig. 2b). In this case, the pseudorandom noise was Gaussian with zero mean and a standard deviation of $0.12 \mathrm{mGal}$. We assume that the density contrast of sediments within the basin varies according to Eq. 1 with $\Delta \rho_{0}=-0.45 \mathrm{gm} / \mathrm{cm}^{3}$, and $\lambda=0.39 \mathrm{~km}^{-1}$. The anomaly shows asymmetric nature across the strike of the structure with large gradient observed over the western margin of the basin (Fig. 2a). The gravity profile along which the interpretation is intended covers the lateral dimensions of the basin and extends farther away to stations resting on the basement. We interpret the gravity anomalies using the proposed inversion technique described in the text to recover the basin structure. For such an inversion, the algorithm had performed 13 iterations before it got terminated as the misfit fell below a predefined allowable error of $10^{-5} \mathrm{mGal}$. The initial structure of the basin estimated by the algorithm is shown in Fig. $2 \mathrm{~b}$ and the corresponding anomaly produced by it in Fig. 2a, respectively. The objective function $J$, reduced drastically from its initial value of 86.8 to 0.0007 at the end of the 3rd iteration and then gradually reaches to almost 0.0 at the end of the 13th iteration (Fig. 2c). The variation of the damping factor, $\delta$, with iteration during the process of inversion is shown in Fig. 2d. The fit between the observed and modeled gravity anomalies (shown in Fig. 2a as a solid line) at the end of the 13th iteration is satisfactory. A maximum error of $0.006 \mathrm{mGal}$ between these 


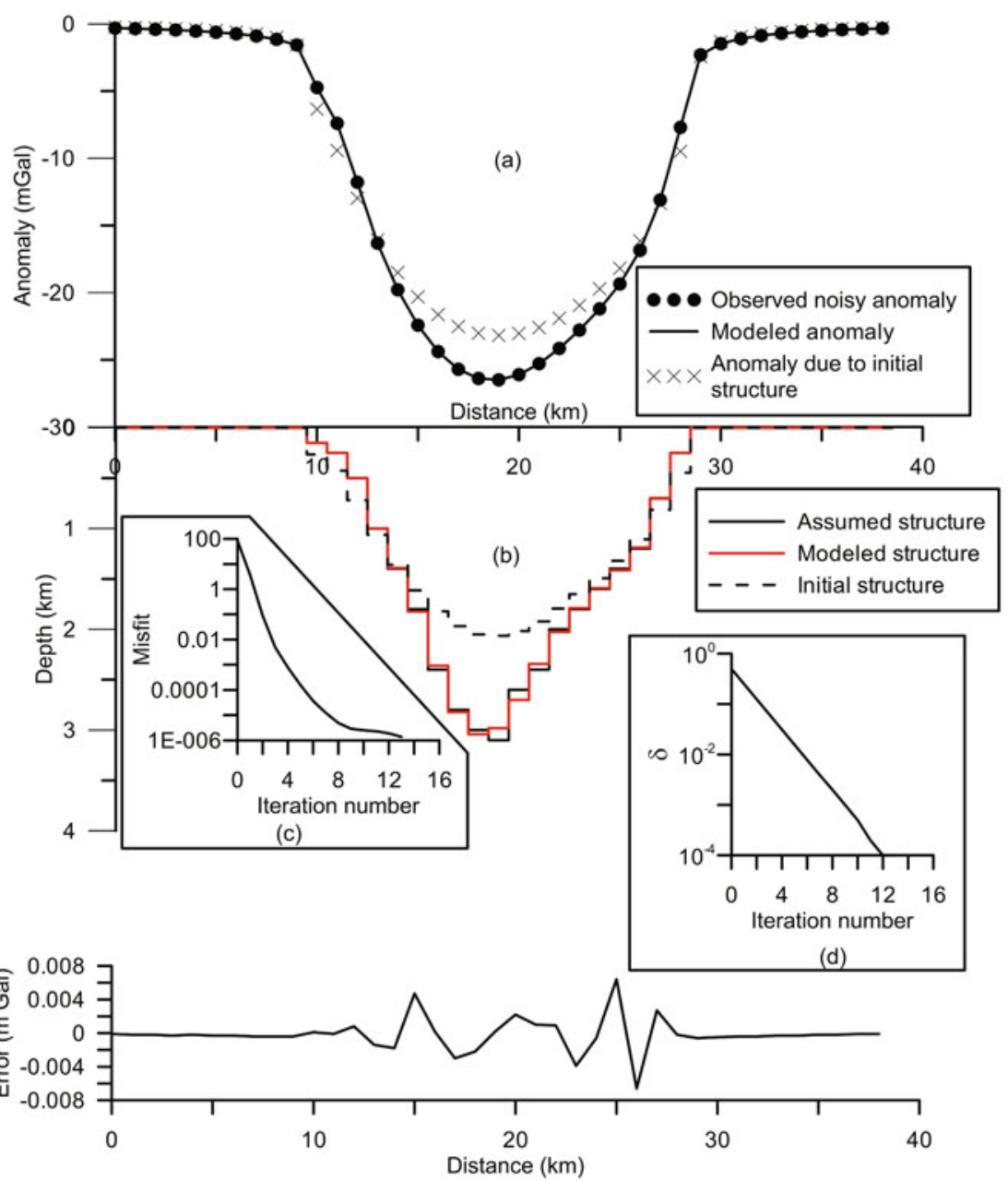

(e)

Fig. 2: (a) Observed and modeled gravity anomalies for initial and estimated depth structures of a synthetic model of a sedimentary basin using exponential density function; (b) assumed, initial and estimated depth structures; (c) variation of objective function with iteration; (d) changes in the damping factor against the iteration number; and (e) error between the observed and modeled gravity anomalies after inversion.

two anomalies is observed at the 25 th $\mathrm{km}$ on the profile (Fig. 2e). The estimated structure subsequent to inversion is shown in Fig. $2 \mathrm{~b}$ along with the assumed structure. No significant changes in the estimated depth are noticed beyond the concluding iteration. 
By and large, the algorithm successfully recovered the structure with a few deviations in and around the depocentre (Fig. 2b). A maximum error of $3.9 \%$ between the assumed and estimated depths is observed at the 20 th $\mathrm{km}$ on the profile (Fig. 2b). Such an error is insignificant considering the presence of noise in the residual signal of the structure.

\subsection{Field example - San Jacinto graben, California}

The San Jacinto graben is bounded by two parallel branches of the San Jacinto fault, and has a northwesterly trend (Cordell 1973). The observed negative gravity anomalies over the graben (Fig. 3a) are attributed to the low density basin fill consisting of Pliocene and Pleistocene detrital sedimentary rocks and Pleistocene and Holocene alluvium. On the basis of seismic refraction data, Fett (1968) had determined the depth to the basement in the center of the graben as $2.4 \mathrm{~km}$. Cordell (1973) had used two exponential density depth models based on Eq. 1 , one with $\Delta \rho_{0}=-0.55 \mathrm{gm} / \mathrm{cm}^{3}$ and $\lambda=$ $0.5 \mathrm{~km}^{-1}$ (shown as solid line in Fig. 3c) and the other with $\Delta \rho_{0}=$ $-0.55 \mathrm{gm} / \mathrm{cm}^{3}$ and $\lambda=1.0 \mathrm{~km}^{-1}$ (shown as dashed line in Fig. 3c) to describe the density contrast-depth data of the graben derived from seismic refraction surveys (shown as step line in Fig. 3c). He demonstrated that the use of former density model in the gravity analysis of the graben had yielded structural solution (shown as dashed line in Fig. 3b) that was consistent with the seismically derived information by Fett (1968).

For the present case, we have interpreted the same gravity data using our inversion technique to decipher its basement configuration. The exponential density model defined with $\Delta \rho_{0}=-0.55 \mathrm{gm} / \mathrm{cm}^{3}$ and $\lambda=0.5 \mathrm{~km}^{-1}$ (Cordell 1973) has been used in the present inversion. We have digitized the anomaly at an interval of $0.322 \mathrm{~km}$ (Fig. 3a) covering a profile length of about $10.0 \mathrm{~km}$ across the graben and subjected the anomalies for inversion. In this case, the algorithm performed 114 iterations (Fig. 3d) before it got terminated. The approximate structure of the graben shown in Fig. 3b was based on the initial depths calculated using Eq. 5. The corresponding gravity response of the initial structure is shown in Fig. 3a. The objective function corresponding to the initial structure was 252.8 (Fig. 3d). The changes in the damping factor, $\delta$, (dashed line) and objective function (solid line) against the iteration number were shown in Fig. 3d. The modeled gravity anomalies at the end of the 114th iteration are shown in Fig. 3a and the inferred structure of the graben in Fig. 3b, respectively. No significant changes are found in estimated depths of the graben subsequent to the concluding iteration. The modeled gravity anomalies and the inferred structure of the graben interpreted by Cordell (1973) are also shown in Fig. 3a and b for comparison. The maximum depth to the basement estimated from present inversion is $2.59 \mathrm{~km}$, 


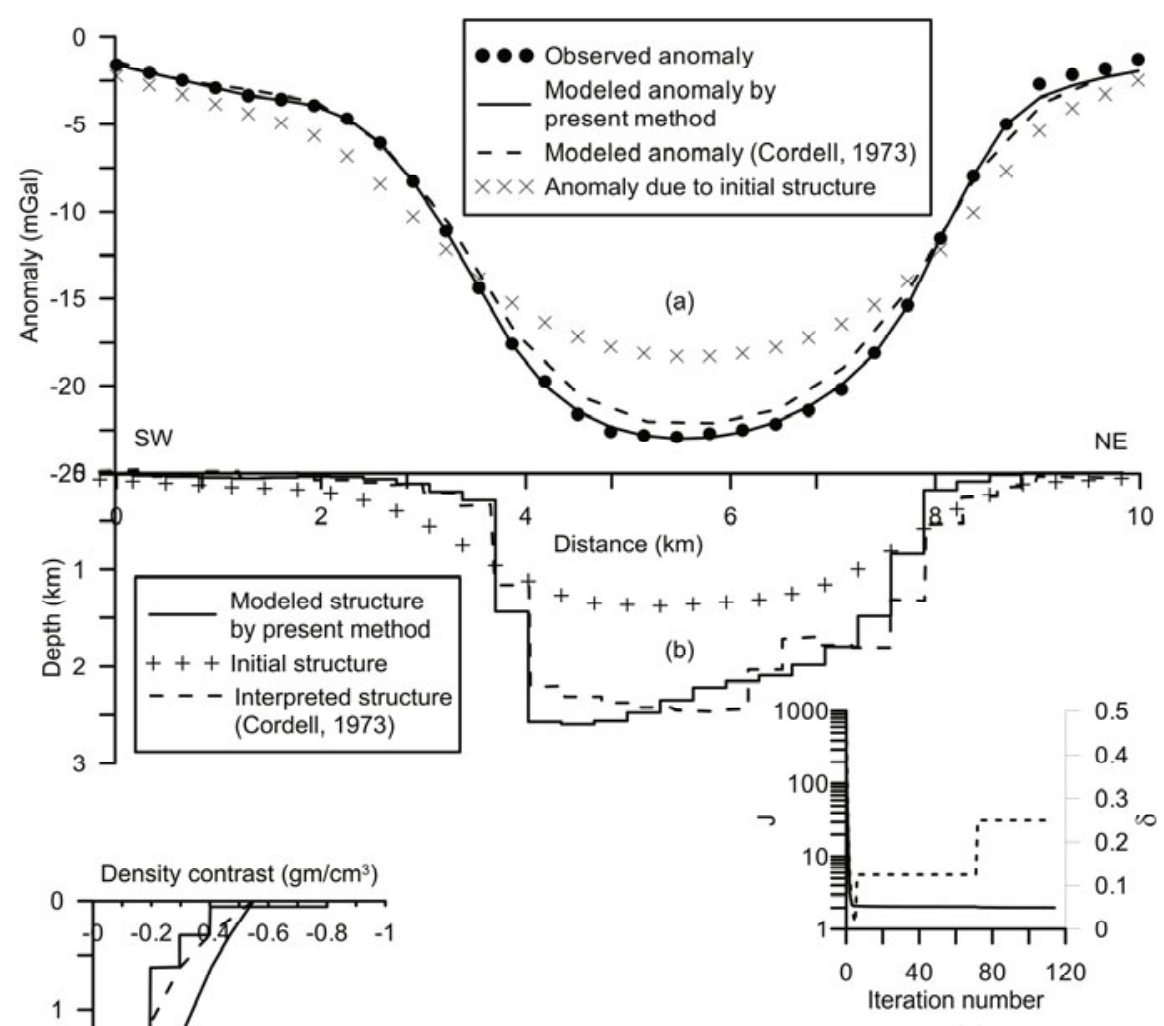

(d)
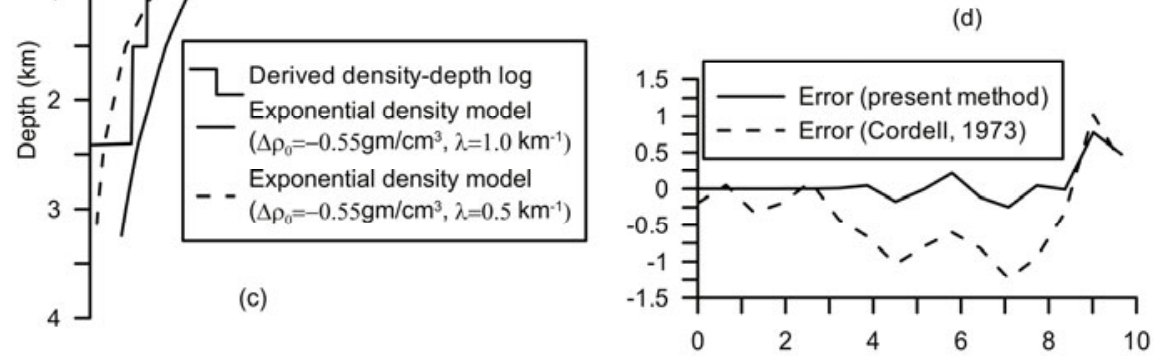

(e)

Fig. 3: (a) Observed and modeled gravity anomalies for initial and estimated depth structures using exponential density model, San Jacinto graben, California; modeled anomalies by Cordell (1973) are also shown; (b) initial and estimated depth structure by the present method; inferred depth model by Cordell (1973) is also shown; (c) predicted density contrast-depth data by Fett (1968) (solid step line) and fitted exponential density models by Cordell (1973); solid line corresponds to $\Delta \rho_{0}=$ $-0.55 \mathrm{gm} / \mathrm{cm}^{3}$ and $\lambda=0.5 \mathrm{~km}^{-1}$ and dashed line corresponds to $\Delta \rho_{0}=$ $-0.55 \mathrm{gm} / \mathrm{cm}^{3}$ and $\lambda=1.0 \mathrm{~km}^{-1}$; (d) variation of objective function and damping factor with iteration number; and (e) error between the observed and modeled gravity anomalies after inversion. 
whereas Cordell (1973) concluded that the maximum thickness of sediments within the graben was at least $2.44 \mathrm{~km}$. The error between the observed and modeled gravity anomalies in each case is shown in Fig. 3e.

By and large, the structural models inferred from the present technique and the one by Cordell (1973) show more or less similar morphological features over the shoulders of the graben. For instance, both models divulge that the graben is bounded by steeply dipping fault system towards southwest and by gently dipping fault system towards northeast. However, the structural model deciphered from our technique reveals a progressive deepening of the basement towards southwest, which however was not repeated in Cordell's (1973) model (Fig. 3b). The basement configuration derived from our analysis appears be more reliable than the one inferred by Cordell (1973) because the modeled gravity anomalies of the structure from the present inversion more closely fit the observed gravity anomalies (Fig. 3a) than the ones realized by Cordell (1973). Further, the error between the observed and modeled gravity anomaly in our case hardly exceeds $0.25 \mathrm{mGal}$ in the central part of the basin, whereas it was more than $-1.1 \mathrm{mGal}$ in Cordell's (1973) interpretation.

\section{CONCLUSIONS}

We develop a space domain based inversion technique using the ridge regression algorithm to analyze the gravity anomalies of sedimentary basins among which the density contrast obeys exponential decrease with depth. We realize forward gravity modeling of sedimentary basins in the space domain using a combination of analytical and numerical approaches. The present technique initiates the structure of a sedimentary basin with an assumption that the observed gravity anomaly at each observation is being generated by an infinite subsurface horizontal slab in which the density contrast decreases exponentially with depth. The proposed inversion generates the initial structure of a sedimentary basin from the observed gravity anomalies and improves the structure in an iterative approach based on the differences between the observed and modeled gravity anomalies until the modeled anomalies closely mimic the observed ones.

The applicability and validity of this inversion technique is demonstrated on both synthetic and real field gravity anomalies. In case of synthetic example pseudorandom noise is added to the residual gravity signal produced by a sedimentary basin prior to inversion. The algorithm almost recovered the basin structure even in the presence of random noise; however insignificant error in the estimated depth is found in around the depocentre of the assumed structure. Further, the observed gravity anomalies over the San Jacinto graben, California have been analyzed using our technique and the estimated structure is compared with the structural model derived originally 
by Cordell (1973). The estimated maximum thickness of the graben from the proposed inversion more or less coincides with the maximum thickness inferred by Fett (1968) and Cordell (1973). However, our deciphered model of the graben modestly deviates from the one by Cordell (1973) in the sense that our model shows progressive deepening of the basement towards the southwest, which was not repeated in later case. The fact that the present inversion technique yielded information that is consistent with the assumed parameters in case of synthetic structure and with available information in case of field example testifies the applicability of the technique.

Acknowledgements. The authors sincerely thank the anonymous reviewers for their critical reviews and feedback to revise the manuscript as presented.

\section{References}

Abbott, R.E., and J.N. Louie (2000), Depth to bedrock using gravimetry in the Reno and Carson City, Nevada, area basins, Geophysics 65, 2, 340-350, DOI: 10.1190/1.1444730.

Abdoh, A., D. Cowan, and M. Pilkington (1990), 3D gravity inversion of the Cheshire basin, Geophys. Prospect. 38, 8, 999-1011, DOI: 10.1111/j.13652478.1990.tb01887.x.

Blakely, R.J. (1995), Potential Theory in Gravity and Magnetic Applications, Cambridge University Press, Cambridge.

Bott, M.H.P. (1960), The use of rapid digital computing methods for direct gravity interpretation of sedimentary basins, Geophys. J. Int. 3, 1, 63-67, DOI: 10.1111/j.1365-246X.1960.tb00065.x.

Chai, Y., and W.J. Hinze (1988), Gravity inversion of an interface above, which the density contrast varies exponentially with depth, Geophysics 53, 6, 837845, DOI: $10.1190 / 1.1442518$.

Chakravarthi, V. (2003), Digitally implemented method for automatic optimization of gravity fields obtained from three-dimensional density interfaces using depth dependent density, US Patent 6615139 B1.

Chakravarthi, V., and B. Ramamma (2013), Gravity anomaly modeling of multiple geological sources having different strike lengths and arbitrary density contrast variations, Near Surf. Geophys. 11, 4, 363-370, DOI: 10.3997/18730604.2013001.

Chakravarthi, V., and N. Sundararajan (2007), Marquardt optimization of gravity anomalies of anticlinal and synclinal structures with prescribed depthdependent density, Geophys. Prospect. 55, 4, 571-587, DOI: 10.1111/ j.1365-2478.2007.00625.x. 
Chappell, A.R., and N.J. Kusznir (2008), Three-dimensional gravity inversion for Moho depth at rifted continental margins incorporating a lithosphere thermal gravity anomaly correction, Geophys. J. Int. 174, 1, 1-13, DOI: 10.1111/j.1365-246X.2008.03803.x.

Cordell, L. (1973), Gravity analysis using an exponential density-depth function San Jacinto Graben, California, Geophysics 38, 4, 684-690, DOI: 10.1190/ 1.1440367.

Cowie, P.A., and G.D. Karner (1990), Gravity effect of sediment compaction: examples from the North Sea and the Rhine Graben, Earth Planet. Sci. Lett. 99, 1-2, 141-153, DOI: 10.1016/0012-821X(90)90078-C.

Fett, J.D. (1968), Geophysical investigation of the San Jacinto Valley, Riverside County, California, M.Sc. Thesis, University of California, Riverside, USA, $87 \mathrm{pp}$.

Filon, L.N.G. (1928), On a quadrature formula for trigonometric integrals, Proc. Roy. Soc. Edinburgh 49, 1, 38-47.

García-Abdeslem, J. (1992), Gravitational attraction of a rectangular prism with depth-dependent density, Geophysics 57, 3, 470-473, DOI: 10.1190/ 1.1443261 .

Gómez-Ortiz, D., R. Tejero-López, R. Babín-Vich, and A. Rivas-Ponce (2005), Crustal density structure in the Spanish Central System derived from gravity data analysis (Central Spain), Tectonophysics 403, 1-4, 131-149, DOI: 10.1016/j.tecto.2005.04.006.

Granser, H. (1987), Three-dimensional interpretation of gravity data from sedimentary basins using an exponential density-depth function, Geophys. Prospect. 35, 9, 1030-1041, DOI: 10.1111/j.1365-2478.1987.tb00858.x.

Guspi, F. (1990), General 2D gravity inversion with density contrast varying with depth, Geoexploration 26, 4, 253-265, DOI: 10.1016/0016-7142(90)90007-F.

Kadima, E., D. Delvaux, S.N. Sebagenzi, L. Tack, and S.M. Kabeya (2011), Structure and geological history of the Congo Basin: an integrated interpretation of gravity, magnetic and reflection seismic data, Basin Res. 23, 5, 499-527, DOI: $10.1111 /$ j.1365-2117.2011.00500.x.

Mantlik, F., and M.J.S. Matias (2010), Interpretation and modeling of regional gravity data of the Aveiro Basin (Northwest Portugal), C. R. Geosci. 342, 11, 823-836, DOI: 10.1016/j.crte.2010.06.005.

Murthy, I.V.R., and D.B. Rao (1979), Gravity anomalies of two-dimensional bodies of irregular cross-section with density contrast varying with depth, Geophysics 44, 9, 1525-1530, DOI: 10.1190/1.1441023.

Murthy, I.V.R., and S.J. Rao (1989), A FORTRAN 77 program for inverting gravity anomalies of two-dimensional basement structures, Comput. Geosci. 15, 7, 1149-1156, DOI: 10.1016/0098-3004(89)90126-X. 
Northwest Geophysical Associates (2004), GM-SYS gravity/magnetic modeling software. User's guide, Version 4.9, Northwest Geophysical Associates, Inc., Corvallis, USA, $101 \mathrm{pp}$.

Rao, C.V., V. Chakravarthi, and M.L. Raju (1994), Forward modelling: Gravity anomalies of two-dimensional bodies of arbitrary shape with hyperbolic and parabolic density functions, Comput. Geosci. 20, 5, 873-880, DOI: 10.1016/0098-3004(94)90118-X.

Rao, D.B., and C.P.V.N.J.M. Rao (1999), Two-dimensional interpretation of gravity anomalies over sedimentary basins with an exponential decrease of density contrast with depth, Proc. Indian Acad. Sci. (Earth Planet. Sci.) 108, 2, 99106, DOI: $10.1007 / \mathrm{BF} 02840488$.

Rao, D.B., M.J. Prakash, and N.R. Babu (1993), Gravity interpretation using Fourier transforms and simple geometrical models with exponential density contrast, Geophysics 58, 8, 1074-1083, DOI: 10.1190/1.1443491.

Singh, B. (2002), Simultaneous computation of gravity and magnetic anomalies resulting from a 2-D object, Geophysics 67, 3, 801-806, DOI: 10.1190/ 1.1484524 .

Won, I.J., and M. Bevis (1987), Computing the gravitational and magnetic anomalies due to a polygon: Algorithms and Fortran subroutines, Geophysics 52, 2, 232-238, DOI: 10.1190/1.1442298.

Zhou, X. (2013), Gravity inversion of 2D bedrock topography for heterogeneous sedimentary basins based on line integral and maximum difference reduction methods, Geophys. Prospect. 61, 1, 220-234, DOI: 10.111/j.13652478.2011.01046.x.

Received 2 October 2013

Received in revised form 13 June 2014

Accepted 22 July 2014 